\title{
Purified Acetaminophen-Glutathione Conjugate Is Able To Induce Oxidative Stress in Rat Liver Mitochondria
}

\author{
T. ROUŠAR ${ }^{1,2}$, E. NÝDLOVÁ ${ }^{1,3}$, P. ČESLA ${ }^{3}$, P. STAŇKOVÁ $^{2}$, O. KUČERA ${ }^{2}$, P. PǍ̌íK ${ }^{4}$, \\ Z. ČERVINKOVÁ ${ }^{2}$
}

${ }^{1}$ Department of Biological and Biochemical Sciences, Faculty of Chemical Technology, University of Pardubice, Pardubice, Czech Republic, ${ }^{2}$ Department of Physiology, Faculty of Medicine in Hradec Králové, Charles University in Prague, Hradec Králové, Czech Republic, ${ }^{3}$ Department of Analytical Chemistry, Faculty of Chemical Technology, University of Pardubice, Pardubice, Czech Republic, ${ }^{4}$ Institute of Organic Chemistry and Technology, Faculty of Chemical Technology, University of Pardubice, Pardubice, Czech Republic

Received March 20, 2012

Accepted July 2, 2012

\section{Summary}

Acetaminophen overdose is the most often cause of acute liver injury. The toxic mechanism is linked to formation of an active metabolite that reacts with glutathione generating acetaminophen-glutathione conjugate (APAP-SG). This compound has been recognized to be non-toxic generally. Our preliminary results showed, however, that APAP-SG could possess a toxic effect too. Therefore, the aim of our study was to prepare, purify and to test possible toxicity of APAP-SG. We prepared APAP-SG using organic synthesis. The conjugate was purified by preparative HPLC and its structure was confirmed using mass spectrometry. Final purity of APAP-SG was $>98 \%$. We estimated a toxic effect of APAP-SG in isolated rat liver mitochondria using a fluorescent ROS probe. We assessed ROS production in presence of complex I or complex II substrates. The increase of ROS-dependent fluorescence in presence of glutamate/malate was $104 \pm 13 \%$ and $130 \pm 10 \%$ in $1 \mathrm{mM}$ and $5 \mathrm{mM}$ APAP-SG, respectively, in comparison with controls. ROS production related to presence of complex II substrate was enhanced 4-times in APAP-SG (5 mM) treated mitochondria (compared to controls). We conclude, we proved our hypothesis that APAP-SG conjugate is able to induce a mitochondrial impairment leading to enhanced ROS production.

\section{Key words}

Acetaminophen toxicity - Glutathione - Acetaminophenglutathione conjugate

\section{Corresponding author}

Tomáš Roušar, Dept. of Biological and Biochemical Sciences, Faculty of Chemical Technology, University of Pardubice, Studentská 273, 53210 Pardubice, Czech Republic. Fax: (+420) 495518 772. E-mail: Tomas.Rousar@upce.cz

\section{Introduction}

Acetaminophen (paracetamol) is a frequently used analgesic and antipyretic. It is a safe drug at therapeutical doses, but its overdose can lead to acute liver injury. The cause of toxicity can be recognized in its metabolic activation. Acetaminophen (APAP) reacts with glucuronate and sulfate in the liver and a small portion of the drug (cca $10 \%$ ) is oxidized to N-acetyl-pbenzoquinone imine (NAPQI) by cytochrome P-450. After overdose, glucuronidation and sulfatation pathways are saturated and most of APAP dose undergoes oxidation. NAPQI reacts with proteins and glutathione thereby an acetaminophen-glutathione conjugate is generated and the glutathione stores are depleted (Jaeschke and Bajt 2006, Mitchell et al. 1973a, Mitchell et al. 1973b). The glutathione depletion leads to increase in ROS production, mitochondrial impairment, cell damage and, finally, liver injury. Although, great attention has been given to detailed description of the mechanism, the exact cause of cell impairment remains unknown.

Acetaminophen-glutathione conjugate (APAP- 
SG) has been considered as a detoxification product generally (Jaeschke and Bajt 2006, Jaeschke et al. 2011). The main cause of hepatotoxicity has been recognized to be related to APAP metabolic activation, the reaction of NAPQI with a variety of compounds and glutathione depletion. Recently, we have found, however, that APAPSG can possess a toxic effect because we proved that glutathione reductase is inhibited in the presence of this compound (Roušar et al. 2010). The additional toxic effect of APAP-SG could be of crucial importance. Thus, the aim of present study was to synthesize de novo and purify APAP-SG, and to assess its possible toxic effect in isolated mitochondria.

\section{Materials and Methods}

\section{Chemicals}

Acetaminophen, GSH, glutathione disulfide (GSSG), glutathione reductase, NADPH, sodium phosphate buffer, potassium phosphate buffer, hydrochloric acid, acetic acid, methanol, and all chemicals used to preparation of MIR05 and mitochondrial isolation medium were purchased from Sigma-Aldrich (USA). The reagents used to the synthesis of APAP-SG conjugate were purchased from Lachema (Czech Republic).

\section{Synthesis of acetaminophen-glutathione conjugate}

The APAP-SG conjugate was synthesized according to the slightly modified method of (Thatcher and Murray 2001); detailed description was published in Roušar et al. (2010). Briefly, acetaminophen was suspended in dry chloroform and a portion of just prepared silver oxide was added. The suspension was stirred, filtered and a solution with NAPQI was obtained. Glutathione (dissolved in $0.1 \mathrm{M}$ sodium phosphate buffer, $\mathrm{pH}$ 7.4) was allowed to react with freshly prepared NAPQI. Reaction mixture was separated and the water was evaporated at $40{ }^{\circ} \mathrm{C}$. The products of the reaction were obtained as a brown-yellow sticky gel.

\section{Purification of acetaminophen-glutathione conjugate}

APAP-SG conjugate was purified using preparative liquid chromatography (pHPLC). A portion (approximately $0.5 \mathrm{~g}$ ) of brown-yellow gel was diluted in distilled water and it was assayed using pHPLC. The conditions of separation were: mobile phase water: methanol : acetic acid (87\%:12\%:1\%, v/v); stationary phase - preparative column Labiospher PSI (250x25 mm; C18; $7 \mu \mathrm{m}$; Labio, Czech Republic). The experiments were performed using isocratic pHPLC system at $25^{\circ} \mathrm{C}$ consisting of preparative pump LCP3102, injection valve equipped with $10 \mathrm{ml}$ external sample loop and UV detector Saphire (all ECOM, Czech Republic). The column was maintained at $25{ }^{\circ} \mathrm{C}$ and the detection wavelength was set at $254 \mathrm{~nm}$. We collected fractions of mobile phase that contained APAP-SG. The mobile phase was evaporated $\left(30^{\circ} \mathrm{C}\right)$ on rotation vacuum evaporator. APAP-SG conjugate was obtained as a solid. Structure of the compound was confirmed using mass spectrometry. Thus the positive-ion electrospray ionization (ESI) mass spectra were measured on the LCQ ion trap analyzer (Thermo Fisher Scientific, Waltham, MA, USA) in the range $\mathrm{m} / \mathrm{z} 50-800$. The samples were dissolved in methanol and analyzed by direct infusion at the flow rate $10 \mu \mathrm{l} / \mathrm{min}$. ESI source was tuned to provide maximum intensity for $[\mathrm{M}+\mathrm{H}]^{+}$ion at $\mathrm{m} / \mathrm{z} 457$, i.e. source voltage was set to $4 \mathrm{kV}$, capillary voltage $24 \mathrm{~V}$, tube lens voltage $0 \mathrm{~V}$, capillary temperature $175^{\circ} \mathrm{C}$ and sheath gas flow rate to 40 units. The tandem mass spectra for precursor $[\mathrm{M}+\mathrm{H}]^{+}$ion were measured in $\mathrm{m} / \mathrm{z}$ range of 125-800 using collision dissociation with $35 \%$ collision energy. Purity of the substance was proved by HPLC/UV at similar detection and separation conditions as in case of pHPLC on system, but the mobile phase flow was $0.7 \mathrm{ml} / \mathrm{min}$ instead of $15 \mathrm{ml} / \mathrm{min}$ and stationary phase was 250x4.5 mm; C18; $5 \mu \mathrm{m}$; Supelco, USA). The separation was performed at $37^{\circ} \mathrm{C}$ on HPLC/UV equipped with LC20ADXR pump, SPD-20A UV detector (Shimadzu, Japan), Rheodyne injection valve with $10 \mu$ l external injection loop (Rheodyne, Oak Harbor, WA, USA) and LCO 102 column thermostat (ECOM, Prague, Czech Republic). In addition, we purified another synthetic product of reaction between glutathione and acetaminophen that consists of two acetaminophen molecules and glutathione - (APAP $)_{2}-\mathrm{SG}$. The structure and purity of this molecule was proved by same protocol as noted above.

\section{Isolation of rat liver mitochondria}

Male Wistar rats (220-250 g) were purchased from Biotest (Czech Republic). The animals had free access to standard laboratory diet (DOS 2B Velaz, Czech Republic) and tap water. All animals received care according to the guidelines set out by the Institutional Animal Use and Care Committee of Charles University, Prague, Czech Republic. Liver mitochondria were isolated by differential centrifugation in a medium 
containing $220 \mathrm{mM}$ mannitol, $70 \mathrm{mM}$ sucrose, $2 \mathrm{mM}$ HEPES, $0.5 \mathrm{mg} / \mathrm{ml}$ bovine serum albumin, $\mathrm{pH} 7.2$ (Bustamante et al. 1977). The liver was homogenized, homogenate was centrifuged (10 min; 800g), and supernatant was centrifuged again (10 min; 8,000g). Mitochondria in the sediment were centrifuged twice in the isolation medium without bovine serum albumin $(2 \mathrm{x}$ $10 \mathrm{~min} ; 10,000 \mathrm{~g})$. The mitochondria in the sediment were diluted in the isolation medium without BSA and were stored on ice until analyzed.

Estimation of GR inhibition through purified synthetic $(A P A P)_{2}-S G$

We used yeast glutathione reductase (from Sacch. cerevisiae; $160 \mathrm{U} / \mathrm{mg}$ prot.). GR activity was determined at $25^{\circ} \mathrm{C}$ in $0.1 \mathrm{M}$ potassium phosphate buffer (pH 7.5) by monitoring of NADPH absorbance decline using well-plate spectrophotometer INFINITE M200 (Tecan, Austria) (Carlberg and Mannervik 1975, Roušar et al. 2010). We tested an effect in (APAP) $)_{2}-S G$ that was diluted in distilled water. The reaction mixture consisted of: $10 \mu \mathrm{l}$ of (APAP) $)_{2}$-SG (1-3 mM), $50 \mu \mathrm{l}$ of GR $(100 \mathrm{mU})$ and $25 \mu \mathrm{l}$ of GSSG (in range of concentrations of 0.1-9.1 mM). Control samples were prepared by identical protocol, the distilled water $(10 \mu \mathrm{l})$ was added instead of APAP-SG conjugate. The measurement was started by addition of NADPH $(25 \mu \mathrm{l}, 1 \mathrm{mM})$ and monitored at $\lambda=340 \mathrm{~nm}$ spectrophotometrically for 10 minutes at $25{ }^{\circ} \mathrm{C}(\mathrm{n}=2)$. ( 1 Unit was defined as an amount of the enzyme which will reduce $1 \mu$ mole of glutathione disulfide per minute at $\mathrm{pH} 7.5$ at $25^{\circ} \mathrm{C}$, using a molar extinction coefficient of $6.22 \times 10^{3}$ for NADPH.) The values in the brackets mean the final concentrations of a compound in well.

\section{Assessment of mitochondrial ROS production}

Isolated rat liver mitochondria were incubated in presence of APAP-SG $(1 \mathrm{mM}, 5 \mathrm{mM})$ and production of reactive oxygen species was determined using a ROS probe during 60 minutes. We used 5-,6-chloromethyl2',7'-dichlorodihydrofluorescein diacetate (CM$\mathrm{H}_{2}$ DCFDA; Molecular Probes, USA) that is nonfluorescent until oxidized by ROS. Mitochondria were incubated in MiR05 medium (EGTA, $0.5 \mathrm{mM}$; $\mathrm{MgCl}_{2} .6 \mathrm{H}_{2} \mathrm{O}, 3 \mathrm{mM}$; K-lactobionate, $60 \mathrm{mM}$; taurine, $20 \mathrm{mM}$; $\mathrm{KH}_{2} \mathrm{PO}_{4}, 10 \mathrm{mM}$; HEPES, $20 \mathrm{mM}$; sucrose, $110 \mathrm{mM}$; pH 7.1). We estimated ROS production related to mitochondrial respiration on complex I and II. To determine ROS production related to complex I activity, we used malate $(2.5 \mathrm{mM})$ and glutamate $(10 \mathrm{mM})$ as substrates. Production of reactive species related to presence of complex II-substrate, succinate $(10 \mathrm{mM})$ was tested; we added also a complex I inhibitor, rotenone $(0.5 \mu \mathrm{M})$. The values in the brackets mean the final concentrations in a well. The final mixture consisted of: $25 \mu \mathrm{l}$ of mitochondrial suspension $\left(0.36 \mathrm{mg}\right.$ prot. $\left.\mathrm{l}^{-1}\right)$, substrates for mitochondrial respiration $(100 \mu \mathrm{l}$, diluted in MIR05 medium) and $25 \mu \mathrm{l}$ of APAP-SG ( $1 \mathrm{mM}$ or $5 \mathrm{mM})$. We used distilled water $(25 \mu \mathrm{l})$ or $5 \mathrm{mM}$ acetaminophen $(25 \mu \mathrm{l})$ instead of APAP-SG to determine base ROS production in controls. Finally, we added $100 \mu \mathrm{l}$ of $\mathrm{CM}-\mathrm{H}_{2}$ DCFDA ( $1 \mu \mathrm{M}$, in PBS). The increase of fluorescence intensity (excitation and emission wavelength, 485 and $535 \mathrm{~nm}$, respectively) was monitored at $37^{\circ} \mathrm{C}$ using fluorescence plate reader Infinite M200 (Tecan, Austria) for 60 minutes. The results were expressed as a difference between fluorescence intensity at the end and at the start of the measurement $(n=3-4)$.

\section{Statistical analysis}

The assays of glutathione reductase activity and mitochondrial ROS production were repeated at least two times with negligible differences among results. The results of ROS production are expressed as the mean \pm SD. The statistical significance was analyzed using one-way ANOVA test followed by Dunnett's post hoc test to compare controls and other groups. (GraphPad Prism 4.03 for Windows, GraphPad Software, USA). $\mathrm{p}<0.05$ was considered as significant.

\section{Results}

We prepared purified APAP-SG conjugate using preparative HPLC. We used mass spectrometry technique and HPLC/UV to confirm its structure (Fig. 1) and purity, respectively (Fig. 2). The purity of prepared APAP-SG was $>98 \%$.

In addition, we prepared another synthetic compound that was produced through the reaction of two acetaminophen molecules and glutathione, (APAP) $)_{2}-\mathrm{SG}$. Its structure and purity was confirmed by MS technique too; purity was $>96 \%$. We suppose, that this compound may occur naturally at low concentration in the cell because NAPQI can react each other or may be hydrolyzed (Coles et al. 1988, Madsen et al. 2007, Miner and Kissinger 1979, Potter and Hinson 1987). Therefore, we estimated possible inhibitory effect of (APAP) $)_{2}$-SG on 
glutathione reductase (GR) activity. We found that (APAP) $)_{2}$-SG was able to decrease glutathione reductase activity. We tested GR activity in presence of a variety of glutathione disulfide concentrations as a natural substrate of GR. The results show that glutathione reductase was inhibited in presence of $2 \mathrm{mM}(\mathrm{APAP})_{2}-\mathrm{SG}$ (Fig. 3) in relation to GSSG concentration. The enzyme activity was inhibited by $37 \%$ and $67 \%$ in presence of $9.1 \mathrm{mM}$ and $0.1 \mathrm{mM}$ GSSG, respectively. Based on our results, we suppose that the inhibitory mechanism of glutathione reductase might be competitive.

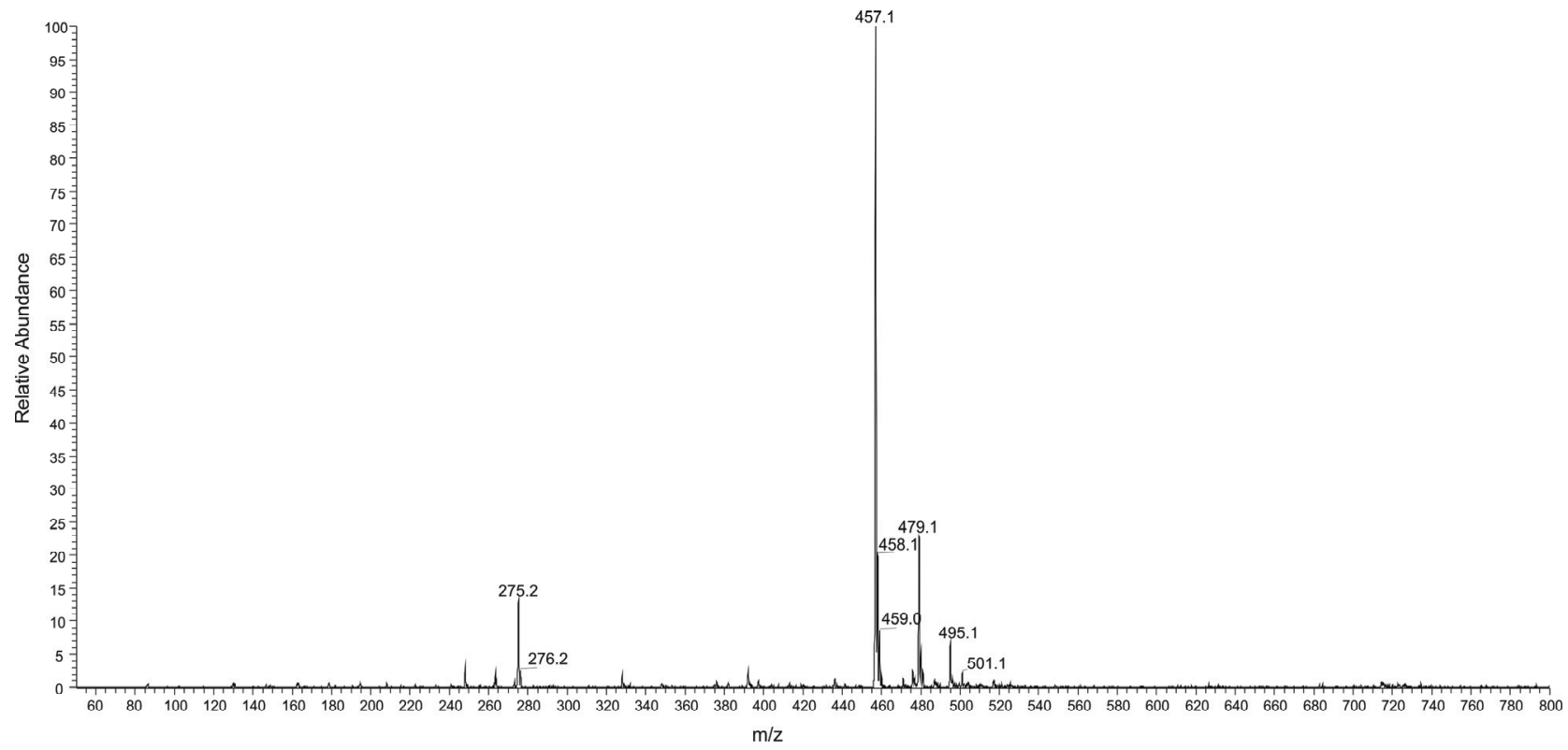

Fig. 1. Tandem mass spectrum of APAP-SG $(0.1 \mathrm{mM})$ dissolved in methanol. Precursor ion $[\mathrm{M}+\mathrm{H}]^{+}$at $\mathrm{m} / \mathrm{z} 457$, collision dissociation energy $35 \%$.

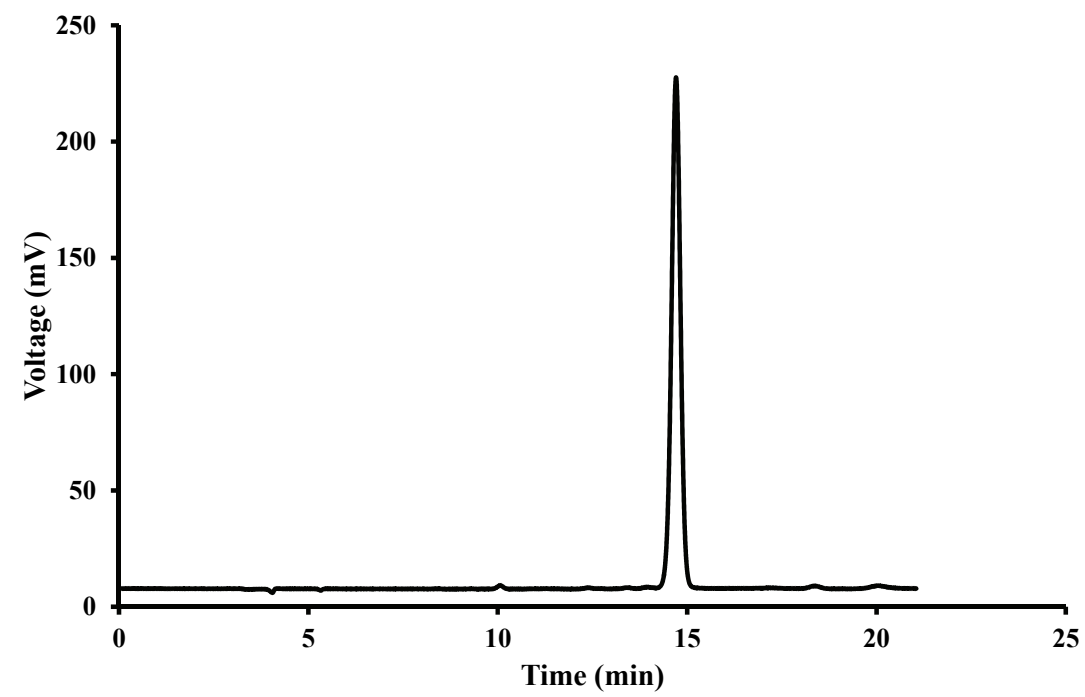

Fig. 2. HPLC/UV analysis of purified APAP-SG (0.2 mM). Mobile phase water : methanol : acetic acid $\quad(87 \%$ : $12 \%: 1 \%, v / v), 0.7 \mathrm{ml} / \mathrm{min}, 37^{\circ} \mathrm{C}$; stationary phase - 250x4.5 mm; C18; $5 \mu \mathrm{m}$ (Supelco, USA); detection at $254 \mathrm{~nm}$.

To determine a direct toxic effect of APAP-SG on mitochondrial function, we estimated mitochondrial ROS production. We assessed ROS production during incubation with substrates for complex I and complex II in presence of $1 \mathrm{mM}$ and $5 \mathrm{mM}$ APAP-SG. We found that ROS production was significantly increased in both experiments. The increase of fluorescence in presence of glutamate and malate (i.e. complex I-related) was $104 \pm 13 \%$ and $130 \pm 10 \%$ in $1 \mathrm{mM}$ and $5 \mathrm{mM}$ APAP-SG, respectively, in comparison with controls (Fig. 4A). Reactive oxygen species production in relation to presence succinate and rotenone was significantly 
increased only in presence of $5 \mathrm{mM}$ APAP-SG where the fluorescence was enhanced by $306 \pm 32 \%$ in comparison to the values of control samples (Fig. 4B). We assessed also mitochondrial ROS production during incubation with acetaminophen $(5 \mathrm{mM})$. Surprisingly, we found that acetaminophen is able to reduce ROS production in presence of both malate/glutamate and succinate/rotenone significantly.

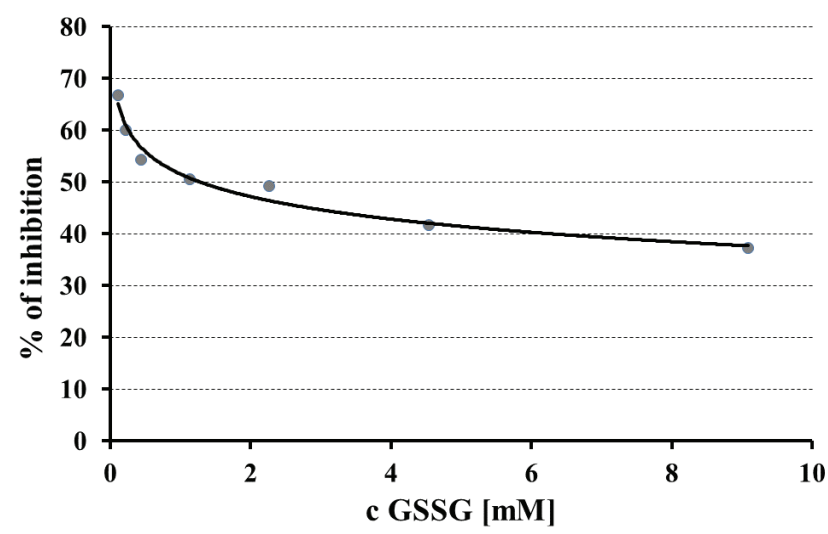

Fig. 3. Inhibitory effect of (APAP) $)_{2}-S G$ on yeast glutathione reductase. The activity of glutathione reductase was measured as decrease of NADPH absorbance at $\lambda=340 \mathrm{~nm}$ during 10 minutes ( $1 \mathrm{U}$ was defined as $\mu \mathrm{mol}$ of GSSG reduced per minute, using a molar extinction coefficient of $6.22 \times 10^{3}$ for NADPH). The results were expressed as percent of inhibition related to control activity at appropriate GSSG concentration in mixture. The range of GSSG concentration was 0.1- 9.1 mM $(n=2-3)$.

\section{Discussion}

Acetaminophen hepatotoxicity has been recognized as a major cause of acute liver injury in USA and UK (Lee 2004). Although the basic mechanism of toxicity was described almost forty years ago (Mitchell et al. 1973a, Mitchell et al. 1973b), the exact cause of cell damage remains unknown (Jaeschke and Bajt 2006, James et al. 2003). Main mechanism of the toxicity has been attributed to NAPQI formation. This highly reactive molecule can bind with - SH groups of proteins thereby APAP-adducts are formed. NAPQI can also react with glutathione producing APAP-SG conjugate. This conjugation might be catalyzed with glutathione-Stransferase (Coles et al. 1988, Henderson and Wolf 2005).

APAP-SG has been considered as a detoxification product generally (Bajt et al. 2004, Jaeschke and Bajt 2006, Jaeschke et al. 2011) since the conjugate protects the cell against the possible toxic effect of NAPQI. On the other hand, there has been described no direct toxic effect of NAPQI in the literature. The pathological processes seem to be related to glutathione depletion followed by pro-oxidative reactions, oxidative stress, mitochondrial impairment and cell death (Jaeschke and Bajt 2006, Jaeschke et al. 2011, Kon et al. 2004).
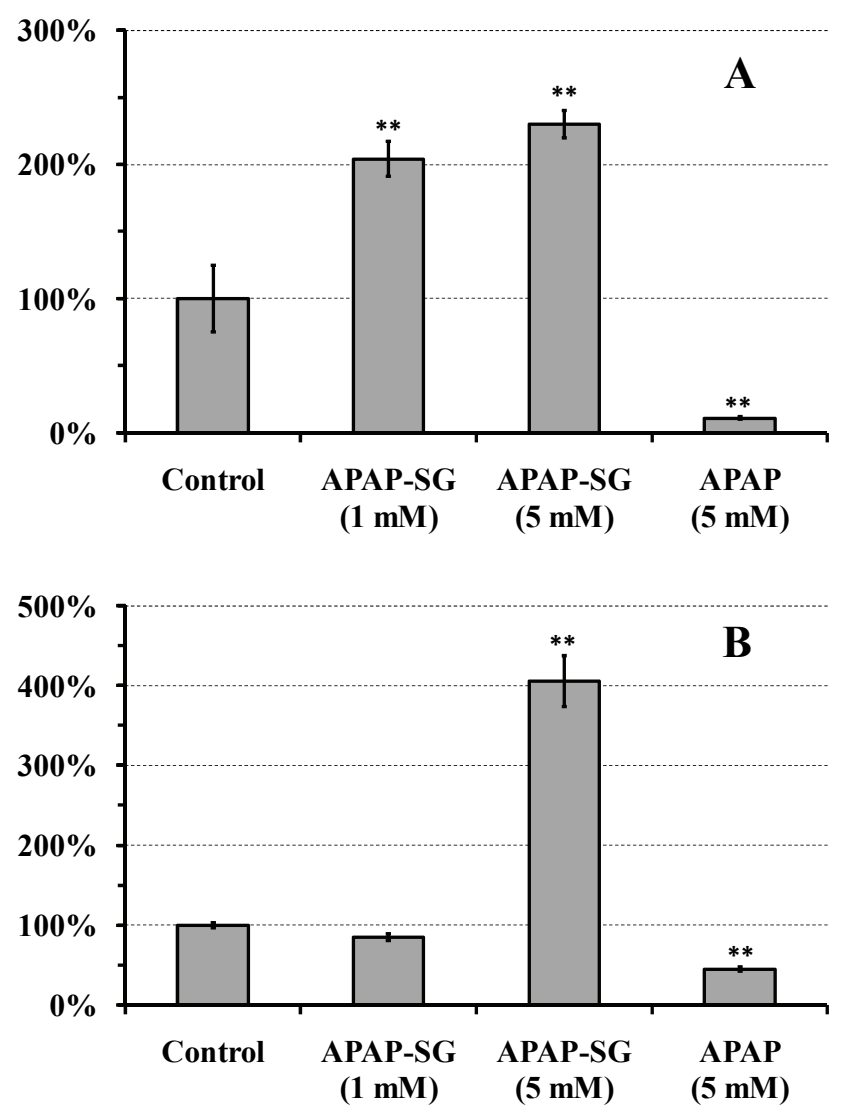

Fig. 4. Mitochondrial ROS production during treatment with APAP-SG. Purified rat liver mitochondria were incubated with APAP-SG $(1 \mathrm{mM}, 5 \mathrm{mM}), \operatorname{APAP}(5 \mathrm{mM})$ or distilled water in controls. The mitochondria were incubated with malate $(2.5 \mathrm{mM})$ + glutamate $(10 \mathrm{mM} ; \mathbf{A})$, or succinate $(10 \mathrm{mM})$ + rotenone $(0.5 \mu \mathrm{M}$; B) for 60 minutes and ROS production was detected. We used a ROS probe $\mathrm{CM}-\mathrm{H}_{2}$ DCFDA that is nonfluorescent until oxidized by ROS. The increase of fluorescence was determined at excitation and emission wavelength, 485 and $535 \mathrm{~nm}$, respectively. The results were expressed as percent increase of ROS-related fluorescence in comparison to control signal $(=100 \%)$. The signal of blank sample (without mitochondria and/or substrates) was under $0.1 \%$ of control signal. Each graph column shows mean $\pm \mathrm{SD}(\mathrm{n}=4)$. $* * \quad \mathrm{p}<0.01$ (compared to control).

Recently, we have formulated a hypothesis that APAP-SG conjugate might possess a toxic effect (Roušar et al. 2010). Therefore, we purified a product of reaction between APAP and GSH by column chromatography and estimated its possible inhibitory effect on glutathione reductase. We found that both rat liver and yeast 
glutathione reductases were inhibited significantly in the presence of APAP-SG (Roušar et al. 2010). Based on our results, we aimed to synthesize, purify and to test APAPSG in other experiments. In addition, we prepared and purified (APAP) ${ }_{2}-\mathrm{SG}$ conjugate by organic synthesis. We found that this compound might be able to inhibit GR activity significantly through competitive mechanism with GSSG. Although this compound has not been proven to occur in cells, it may appear in cells due to high reactivity of NAPQI and following hydrolysis (Coles et al. 1988, Madsen et al. 2007, Miner and Kissinger 1979, Potter and Hinson 1987).

In this study, we prepared APAP-SG by organic synthesis and we purified it using preparative HPLC. We confirmed the structure by mass spectrometry and tested the purity of prepared APAP-SG powder. The final purity of APAP-SG was over $98 \%$. We used this molecule to induce oxidative stress in rat liver mitochondria. We found that mitochondria produced significantly higher levels of reactive oxygen species during incubation with APAP-SG in presence of metabolic substrates for both complex I and complex II. In addition, we found that complex I-associated production of ROS is increased in relation to APAP-SG dose. ROS production in presence of succinate cannot be caused through direct ROS production on complex II. However, there is number of complex I-independent mechanisms that might explain this outcome (Murphy 2009). Interestingly, we found out that ROS production in presence of substrates for both complexes was significantly reduced in APAP treated mitochondria. Mitochondria are not able to metabolize acetaminophen to NAPQI because they lack P450 cytochrome. Therefore, our finding that ROS production is reduced in APAP presence may be caused by unknown mechanism.

Here described APAP-SG-associated production of ROS may be of large importance. We used APAP-SG concentrations (1-5 mM) that can be similar to APAP-SG levels occurring in hepatocytes after overdose. Reduced glutathione occurs in millimolar levels in cells; especially in the liver, glutathione levels occur at 6-7 $\mathrm{mM} \mathrm{GSH} \mathrm{(Lu}$ 1999, Tateishi et al. 1977). After overdose, the most of APAP is oxidized on cytochrome P450 and generated NAPQI reacts with GSH (Mitchell et al. 1973a, Mitchell et al. 1973b). Since the glutathione stores are decreased to levels under $1 \mathrm{mM} \mathrm{GSH}$ very quickly (Mitchell et al. 1973b), most of glutathione can be depleted due to reaction with NAPQI. Thus, the concentration of APAPSG may occur at millimolar levels.
Our results showing the importance of APAPSG related toxicity can be also supported by studies of other authors. Henderson and Wolff (Henderson et al. 2000) tested acetaminophen toxicity in glutathione-Stransferase pi knockout mice. GST-pi catalyzes conjugation of GSH with NAPQI so that GST-pi null mice could have lower levels of APAP-SG in hepatocytes. The authors hypothesized that GST-pi knockout mice should be more susceptible to APAP toxicity because these animals had higher levels of NAPQI that reacts with cellular biomolecules instead of GSH (Henderson et al. 2000). Their results showed, however, that the GST-pi null animals were significantly more resistant to APAP toxicity than control animals with normal GST-pi activity. These surprising results might be explained concerning our outcomes because the production of APAP-SG was decreased in GST-pi null mice in comparison with control animals. Thus, lower levels of APAP-SG likely did not cause as severe injury as in control animals.

\section{Conclusion}

The obtained results support our hypothesis about possible pathological role of APAP-SG conjugate. Our results are supported by outcomes of other studies. Overall, APAP-SG conjugate might play one of the crucial roles in APAP hepatotoxicity and we would like to focus on description of its toxic effect in future.

\section{Conflict of Interest}

There is no conflict of interest.

\section{Acknowledgements}

This work was supported by the programme PRVOUK P37/02. The authors would like to thank to a company Ratiochem, s.r.o. for cooperation.

\section{Abbreviations}

APAP, acetaminophen; APAP-SG, acetaminophenglutathione conjugate; (APAP) ${ }_{2}$-SG, acetaminophenacetaminophen-glutathione conjugate; BSA, bovine serum albumin; CM- $\mathrm{H}_{2}$ DCFDA, 5-,6-chloromethyl-2', 7'dichlorodihydrofluorescein diacetate; GSH, glutathione (reduced form); GSSG, glutathione disulfide; GST-pi, glutathione-S-transferase pi; GR, glutathione reductase; HPLC/UV, high performance liquid chromatography with UV detection; NADPH, nicotinamide adenine dinucleotide phosphate; NAPQI, N-acetyl-p- 
benzoquinone imine; pHPLC, preparative high oxygen species. performance liquid chromatography; ROS, reactive

\section{References}

BAJT ML, KNIGHT TR, LEMASTERS JJ, JAESCHKE H: Acetaminophen-induced oxidant stress and cell injury in cultured mouse hepatocytes: protection by N-acetyl cysteine. Toxicol Sci 80: 343-349, 2004.

BUSTAMANTE E, SOPER JW, PEDERSEN PL: A high-yield preparative method for isolation of rat liver mitochondria. Anal Biochem 80: 401-408, 1977.

CARLBERG I, MANNERVIK B: Purification and characterization of the flavoenzyme glutathione reductase from rat liver. J Biol Chem 250: 5475-5480, 1975.

COLES B, WILSON I, WARDMAN P, HINSON JA, NELSON SD, KETTERER B: The spontaneous and enzymatic reaction of N-acetyl-p-benzoquinonimine with glutathione: a stopped-flow kinetic study. Arch Biochem Biophys 264: 253-260, 1988.

HENDERSON CJ, WOLF CR: Disruption of the glutathione transferase pi class genes. Methods Enzymol 401: 116$135,2005$.

HENDERSON CJ, WOLF CR, KITTERINGHAM N, POWELL H, OTTO D, PARK BK: Increased resistance to acetaminophen hepatotoxicity in mice lacking glutathione S-transferase Pi. Proc Natl Acad Sci U S A 97: 12741-12745, 2000.

JAESCHKE H, BAJT ML: Intracellular signaling mechanisms of acetaminophen-induced liver cell death. Toxicol Sci 89: 31-41, 2006.

JAESCHKE H, MCGILL MR, WILLIAMS CD, RAMACHANDRAN A: Current issues with acetaminophen hepatotoxicity - a clinically relevant model to test the efficacy of natural products. Life Sci 88: 737-745, 2011.

JAMES LP, MAYEUX PR, HINSON JA: Acetaminophen-induced hepatotoxicity. Drug Metab Dispos 31: 1499-1506, 2003.

KON K, KIM JS, JAESCHKE H, LEMASTERS JJ: Mitochondrial permeability transition in acetaminophen-induced necrosis and apoptosis of cultured mouse hepatocytes. Hepatology 40: 1170-1179, 2004.

LEE WM: Acetaminophen and the U.S. Acute Liver Failure Study Group: lowering the risks of hepatic failure. Hepatology 40: 6-9, 2004.

LU SC: Regulation of hepatic glutathione synthesis: current concepts and controversies. FASEB J 13: 1169-1183, 1999.

MADSEN KG, OLSEN J, SKONBERG C, HANSEN SH, JURVA U: Development and evaluation of an electrochemical method for studying reactive phase-I metabolites: correlation to in vitro drug metabolism. Chem Res Toxicol 20: 821-831, 2007.

MINER DJ, KISSINGER PT: Evidence for the involvement of N-acetyl-p-quinoneimine in acetaminophen metabolism. Biochem Pharmacol 28: 3285-3290, 1979.

MITCHELL JR, JOLLOW DJ, POTTER WZ, DAVIS DC, GILLETTE JR, BRODIE BB: Acetaminophen-induced hepatic necrosis. I. Role of drug metabolism. J Pharmacol Exp Ther 187: 185-194, 1973a.

MITCHELL JR, JOLLOW DJ, POTTER WZ, GILLETTE JR, BRODIE BB: Acetaminophen-induced hepatic necrosis. IV. Protective role of glutathione. J Pharmacol Exp Ther 187: 211-217, $1973 \mathrm{~b}$.

MURPHY MP: How mitochondria produce reactive oxygen species. Biochem J 417: 1-13, 2009.

POTTER DW, HINSON JA: Mechanisms of acetaminophen oxidation to N-acetyl-P-benzoquinone imine by horseradish peroxidase and cytochrome P-450. J Biol Chem 262: 966-973, 1987.

ROUŠAR T, PAŘÍK P, KUČERA O, BARTOŠ M, ČERVINKOVÁ Z: Glutathione reductase is inhibited by acetaminophen-glutathione conjugate in vitro. Physiol Res 59: 225-232, 2010.

TATEISHI N, HIGASHI T, NARUSE A, NAKASHIMA K, SHIOZAKI H: Rat liver glutathione: possible role as a reservoir of cysteine. J Nutr 107: 51-60, 1977.

THATCHER NJ, MURRAY S: Analysis of the glutathione conjugate of paracetamol in human liver microsomal fraction by liquid chromatography mass spectrometry. Biomed Chromatogr 15: 374-378, 2001. 